\title{
Produtividade e classificação de brócolos para indústria em função da adubação nitrogenada e potássica e dos espaçamentos entre plantas
}

\author{
Arthur B Cecílio Filho ${ }^{1}$; Aparecido Alecio Schiavon Júnior ${ }^{2}$; Juan Waldir M Cortez ${ }^{1}$ \\ ${ }^{1}$ UNESP-FCAV, Dep ${ }^{\text {to }}$ Prod. Vegetal, Rodov. Prof. Paulo D. Castellane s/n, 14884-900 Jaboticabal-SP; rutra@fcav.unesp.br; invic64@ \\ hotmail.com; ${ }^{2}$ Syngenta Seeds Ltda. Av. das Nações Unidas 18.001, 4ªndar, 04795-900 São Paulo-SP; alecio.schiavon@syngenta.com
}

\begin{abstract}
RESUMO
No município de Itatiba-SP, no período de março a julho de 2007, foi realizado um experimento com o objetivo de avaliar doses de nitrogênio e potássio e espaçamentos entre plantas na produtividade e classificação de brócolos para indústria. $\mathrm{O}$ experimento foi instalado sob delineamento de blocos ao acaso, com os tratamentos em esquema fatorial $5 \times 4$, e três repetições, sendo avaliadas as doses de nitrogênio-potássio: 105-105; 157,5-157,5; 210-210; 262,5-262,5 e 315-315 $\mathrm{kg} \mathrm{ha}^{-1}$ de $\mathrm{N}_{\text {e }} \mathrm{K}_{2} \mathrm{O}$ e os espaçamentos entre plantas: 0,$2 ; 0,3 ; 0,4$ e $0,5 \mathrm{~m}$. O espaçamento entre linhas foi de 0,8 $\mathrm{m}$. Foram avaliados o teor foliar de nitrogênio $(\mathrm{TN})$ e de potássio $(\mathrm{TP})$, produtividade de inflorescência (PI), produtividade de floretes $(\mathrm{PF})$, rendimento industrial (RI) e classificação dos floretes com base em seu comprimento: PF5 ( $\leq 5 \mathrm{~cm})$; PF57 ( $>5 \mathrm{e} \leq 7 \mathrm{~cm})$; PF79 ( $>7 \mathrm{e} \leq 9 \mathrm{~cm})$ e PF9 ( $>9 \mathrm{~cm})$. Maiores espaçamentos entre plantas proporcionaram maior TN e menores PI e PF. As doses NK e os espaçamentos entre plantas não influenciaram significativamente o TP e o RI. As maiores produtividades de inflorescências $\left(22,08\right.$ t ha $\left.^{-1}\right)$ e de floretes $\left(17,09 \mathrm{tha}^{-1}\right)$ foram obtidas com 0,20 m entre plantas e $315 \mathrm{~kg} \mathrm{ha}^{-1}$ de $\mathrm{N}$ e de $\mathrm{K}_{2} \mathrm{O}$. Maior percentagem de floretes na classe PF5 foi obtida no menor espaçamento entre plantas. As doses NK e os espaçamentos entre plantas não influenciaram as percentagens de floretes nas classes PF79 e PF9.
\end{abstract}

Palavras-chave: Brassica oleracea var. italica, nitrogênio, potássio, população de plantas, rendimento industrial.

\begin{abstract}
Yield and classification of broccoli depending on fertilizing and spacing between plants

In Itatiba, São Paulo state, Brazil, during the period between March and July of 2007, an experiment was carried out aiming to appraise the effect of doses of nitrogen and potassium (105-105, $157.5-157.5,210-210,262.5-262.5$ and $315-315 \mathrm{~kg} \mathrm{ha}^{-1} \mathrm{~N}$ and $\mathrm{K}_{2} \mathrm{O}$ ) and spacing between plants $(0.2,0.3,0.4$ and $0.5 \mathrm{~m})$ upon the yield of broccoli and their classification. The experimental design was carried out in a randomized block, with the treatments in a $5 \mathrm{x} 4$ factorial scheme and three replications. Rows of plants were $0.8 \mathrm{~m}$ apart. The following characteristics were evaluated: foliar content of nitrogen $(\mathrm{N})$ and potassium $(\mathrm{K})$, head yield $(\mathrm{HY})$, florets yield (FY), industrial yield (IY) and classification of florets based on length: FY5 $(\leq 5 \mathrm{~cm})$; FY57 ( $>5$ and $\leq 7 \mathrm{~cm}) ; \mathrm{FY} 79(>7$ and $\leq 9 \mathrm{~cm})$ and FY9 $(>9$ $\mathrm{cm})$. The largest spacing between plants provided the highest $\mathrm{N}$, but HY and FY were reduced. The NK dose and spacing between plants did not influence significantly the level of $\mathrm{K}$ and IY. The maximal HY (22.08 tha-1) and FY (17.09 $\left.\mathrm{t} \mathrm{ha}^{-1}\right)$ were obtained with $0.20 \mathrm{~m}$ between plants and $150 \% \mathrm{NK}$ dose $\left(315 \mathrm{~kg} \mathrm{ha}^{-1}\right.$ of $\mathrm{N}$ and $\left.\mathrm{K}_{2} \mathrm{O}\right)$. The higher percentage of florets in FY5 was obtained with smaller spacing between plants and NK dose and spacing did not influence significantly the percentage of florets with lengths greater than $7 \mathrm{~cm}$.
\end{abstract}

Keywords: Brassica oleracea var. italica, nitrogen, potassium, plant population, industrial yield.

(Recebido para publicação em 22 de novembro de 2010; aceito em 12 de setembro de 2011) (Received on November 22, 2010; accepted on September 12, 2011)

$\mathrm{O}$ cultivo do brócolo está mais concentrado na região Centro-Sul do Brasil com destaques para o Distrito Federal, Rio Grande do Sul, Paraná e São Paulo. A comercialização, no Brasil, tem sido feita in natura ou processada, sendo esta principalmente na forma congelada, que possui vantagens em relação à primeira, dentre elas a de proporcionar a oferta regular do produto ao longo do ano, em quantidade e preço. As principais indústrias brasileiras estão localizadas nos estados de São Paulo e Rio Grande do Sul.

Entre 62 espécies de hortaliças, o brócolo ocupa o primeiro lugar no teor de vitaminas $\mathrm{A}_{\text {e }} \mathrm{B}_{2}$ e dos minerais cálcio e ferro (Luengo et al., 2007) e tem sido demonstrado o seu efeito na diminuição do risco de câncer (Steinkellner et al., 2001; Kristal \& Lampe, 2002; Johnson, 2002). Entre os dois grupos de brócolos, o ramoso e o de cabeça, esse tem melhor qualidade para congelamento e deve ganhar preferência para cultivo em relação ao brócolo ramoso. Entretanto, por ser de cultivo recente no Brasil, há pequena disponibilidade de informações técnicas para o manejo cultural do brócolo de cabeça, demandando estudos, dentre outros fatores de produção, sobre a nutrição, a adubação e a densidade populacional.

Relativo à parte nutricional e de fertilização, o nitrogênio e o potássio são demandados em grandes quantidades pelo brócolo (Seabra Junior, 2005).

Arjona \& Greig (1984) estudaram a influência de espaçamentos e doses de nitrogênio em brócolo e observaram que o número de inflorescências grandes ( $>5 \mathrm{~cm}$ de diâmetro) e a massa da inflorescência aumentaram até $112 \mathrm{~kg}$ ha $^{-1}$ de nitrogênio, dentro do intervalo de 0 a $168 \mathrm{~kg} \mathrm{ha}^{-1}$. Resultados semelhantes foram obtidos por Gorski \& Armstrong (1985) e Everaarts (1994).

O potássio é muito importante para a obtenção de uma inflorescência de qualidade, sendo especialmente importante sua disponibilidade a partir da formação da cabeça. Mais de 60 enzimas necessitam de um cátion monovalente para sua ativação e, para a grande maioria, o po- 
tássio é esse cátion. Também, participa no transporte através das membranas, neutralização de ânions e manutenção do potencial osmótico. Estas funções fazem com que o $\mathrm{K}$ seja o cátion mais abundante no vegetal (Epstein \& Bloom, 2006).

O espaçamento de plantio de brócolo é estabelecido, principalmente, em razão do segmento de mercado, ou seja, para consumo in natura ou processamento. Tradicionalmente, tem-se adotado o espaçamento de $0,8 \times 0,5 \mathrm{~m}$, ou seja, 25.000 plantas $^{-1}$, quando destinado ao mercado in natura. Com vistas ao mercado de processamento, uma população de 31.250 plantas ha $^{-1}$ é mais utilizada, correspondendo ao espaçamento de 0,8 x 0,4 m. No estado da Califórnia, Estados Unidos, responsável por 90\% da quantidade produzida naquele país, a densidade populacional dos cultivos de brócolos pode chegar a 127.000 plantas/ ha (LeStrange et al., 1996). Porém, em Oklahoma, Kahn et al. (2004) recomendam o plantio em filas duplas com uma população de 64.600 a 95.700 plantas $\mathrm{ha}^{-1}$. Outros pesquisadores, também norte-americanos, recomendam 34.600 a 51.900 plantas ha-1 (Davis, 1998) e entre 73.805 a 107.635 plantas $\mathrm{ha}^{-1}$ (Sanders, 2001). As razões para as diferentes propostas não foram apresentadas mas, provavelmente, se devem às diferentes regiões produtoras e cultivares empregadas. Em geral, cultivos mais adensados têm maiores produtividades, embora com redução na massa e diâmetro da inflorescência (Crisp et al., 1986; Paschold, 1986; Francescangeli et al., 2006). Certamente, alterações na densidade populacional correspondem a modificações na demanda por nutrientes.

O trabalho teve como objetivo avaliar a produtividade, o rendimento industrial e a classificação de floretes de brócolos em função do espaçamento entre plantas e dose de nitrogênio e potássio.

\section{MATERIAL E MÉTODOS}

O experimento foi realizado em área experimental da Syngenta Seeds Ltda., localizada no município de Itatiba-SP (2301'15'S, 4645'00'W, $755 \mathrm{~m}$ de altitude).
$\mathrm{O}$ experimento foi instalado sob delineamento de blocos ao acaso, em esquema fatorial $5 \times 4$, com três repetições. Os 20 tratamentos corresponderam à combinação dos fatores espaçamentos entre plantas $(0,2 ; 0,3 ; 0,4$ e $0,5 \mathrm{~m})$ e doses de nitrogênio-potássio (105-105; $157,5-157,5 ; 210-210 ; 262,5-262,5$ e 315-315 kg ha-1 de $\mathrm{N}$ e de $\mathrm{K}_{2} \mathrm{O}$ ). Essas doses corresponderam a 50, 75, 100, 125 e $150 \%$ das doses de $210 \mathrm{~kg} \mathrm{ha}^{-1}$ de $\mathrm{N}$ e $210 \mathrm{~kg} \mathrm{ha}^{-1} \mathrm{de} \mathrm{K}_{2} \mathrm{O}$, as quais foram obtidas na recomendação de Trani et al. (1997) para a cultura do brócolo, e de acordo com o teor de $\mathrm{K}$ na análise do solo. O solo foi classificado como Latossolo Vermelho Amarelo e apresentou, na camada de 0 a $20 \mathrm{~cm}, \mathrm{pH}=5,7 ; \mathrm{MO}=$ $16 \mathrm{~g} \mathrm{dm}^{-3} ; \mathrm{P}=113 \mathrm{~g} \mathrm{dm}^{-3} ; 4,9 ; 34 ; 12 ; 18$ e $0 \mathrm{mmol}_{\mathrm{c}} \mathrm{dm}^{-3}$ de $\mathrm{K}, \mathrm{Ca}, \mathrm{Mg}, \mathrm{H}+\mathrm{Al}$ e $\mathrm{Al}$, respectivamente; $\mathrm{V}=74,4 \% ; 27$; 0,$91 ; 3,2 \mathrm{~g} \mathrm{dm}^{-3}$ de $\mathrm{S}, \mathrm{B}$ e Zn, respectivamente; 51 e $68 \mathrm{mmol}_{\mathrm{c}} \mathrm{dm}^{-3}$ de SB e CTC, respectivamente.

Cada parcela foi constituída por 24 plantas, distribuídas em três linhas com oito plantas. Foram consideradas plantas úteis para avaliação das características somente as seis plantas centrais da linha central das parcelas.

O preparo do solo foi realizado com aração e duas gradagens e os sulcos abertos com $20 \mathrm{~cm}$ de profundidade. A calagem e as adubações com fósforo (superfosfato simples), boro (ácido bórico) e foliares de boro e molibdênio (molibdato de amônio) foram realizadas conforme recomendação de Trani et al. (1997) para a cultura do brócolo.

O nitrogênio foi fornecido pelo fertilizante uréia e o potássio pelo cloreto de potássio. No plantio, foram aplicados $28,6 \%$ da dose de cada tratamento e o restante $(71,4 \%)$ aplicado em cobertura, parcelado aos 15, 30, 45 e 60 dias após o transplante, épocas recomendadas por Trani et al. (1997). Do total em cobertura, foram aplicados 20\%, 30\%, 30\% e $20 \%$ na primeira, segunda, terceira e quarta épocas, respectivamente.

Fez-se a semeadura da cultivar Mônaco, híbrido $\mathrm{F}_{1}$, da Syngenta Seeds Ltda., no dia 20 de março de 2007, em bandejas de 200 células. O transplante foi realizado quando as mudas apresentavam quatro folhas, aos 30 dias após a semeadura. $\mathrm{O}$ espaçamento entre as linhas foi de $0,8 \mathrm{~m}$. O controle de plantas daninhas foi feito por meio de capinas manuais. Os controles de pragas (traça das crucíferas e curuquerê) e doenças (podridão negra e míldio) foram feitos de maneira preventiva com aplicação de deltametrina, acefato, methomyl, oxicloreto de cobre+mancozeb, mancozeb. A irrigação foi por aspersão, com irrigações diárias até 30 dias após o transplante, e a cada dois dias até a colheita.

Foram avaliados os teores foliares de $\mathrm{N}$ e $\mathrm{K}$, com amostragem da folha recém desenvolvida da planta, quando visualizada a inflorescência, conforme recomendação de Trani \& Raij (1997). Também, foram avaliadas as produtividades de inflorescência (PI) e produtividade de florestes (PF) e o rendimento industrial, correspondente à razão entre PF e PI. Os floretes foram destacados do caule e medidos o comprimento (base ao topo do florete) e avaliadas a produtividade total de floretes ( $\%$ ) nas classes PF5 (florete com comprimento $\leq 5 \mathrm{~cm}$ ); PF57 ( $>5$ e $\leq 7 \mathrm{~cm}) ; \operatorname{PF} 79$ ( $>7$ e $\leq 9 \mathrm{~cm})$ e PF9 $(>9 \mathrm{~cm})$. Estas classes foram propostas baseando-se nas empresas ATI-GEL Vegetais Congelados e NUTRIZ Indústria e Comércio de Alimentos, as quais adotam como tamanho padrão de floretes os de comprimento entre 7 e 10 $\mathrm{cm}$ e 4 a $6 \mathrm{~cm}$, respectivamente.

Foi realizada análise de variância (teste F), segundo o delineamento proposto, utilizando-se o Programa SAS. Para melhor interpretação do efeito conjunto dos fatores densidade de plantio e dose NK, independentemente de ter havido interação significativa dos fatores na análise de variância, foi realizada a análise de superfície de resposta polinomial quadrática. Quando este modelo apresentou ajuste significativo $(\mathrm{p}<0,05)$, a superfície de resposta foi utilizada para interpretar e discutir o efeito dos fatores. Quando não significativa, utilizou-se o estudo de regressão polinomial até segundo grau. A equação da regressão polinomial múltipla na superfície de resposta de segunda ordem seguiu o modelo: $\mathrm{Y}=\mathrm{b}_{0}+\mathrm{b}_{1}($ dose $\mathrm{NK})$ $+\mathrm{b}_{2}$ (espaçamento $)+\mathrm{b}_{3}(\text { dose } \mathrm{NK})^{2}+$ $\mathrm{b}_{4}($ dose $\mathrm{NK}) *($ espaçamento $)+\mathrm{b}_{5}$ (espaçamento $)^{2}$, sendo: $b_{0}=$ intercepto; $b_{1}=$ coeficiente linear para a dose $\mathrm{NK} ; \mathrm{b}_{2}=$ 
coeficiente linear para o espaçamento; $\mathrm{b}_{3}=$ coeficiente quadrático para a dose $\mathrm{NK} ; b_{4}=$ coeficiente da interação entre os fatores dose NK e o espaçamento; $\mathrm{b}_{5}=$ coeficiente quadrático para o espaçamento.

\section{RESULTADOS E DISCUSSÃO}

O teor de nitrogênio na folha diagnóstica foi influenciado significativamente somente pela dose NK. Porém, houve ajuste significativo da superfície de resposta em função da interação dose NK e espaçamento (Figura 1).

Maior teor de $\mathrm{N}$ na folha diagnóstica $\left(43,2 \mathrm{~g} \mathrm{~kg}^{-1}\right)$ foi observado com $0,5 \mathrm{~m}$ entre plantas e $125 \%$ da dose NK, que equivale a $262,5 \mathrm{~kg} \mathrm{ha}^{-1}$ de $\mathrm{N}$ e de $\mathrm{K}_{2} \mathrm{O}$. $\mathrm{O}$ teor de $\mathrm{N}$ foi incrementado com o aumento na dose NK, visto que sob menor espaçamento, $0,2 \mathrm{~m}$ entre plantas, foram constatadas plantas com teores entre 42,7 e $43 \mathrm{~g} \mathrm{~kg}^{-1}$, quando a adubação foi realizada com $125 \%$ a $150 \%$ de NK (Figura 1). Trani \& Raij (1997) consideram teores de $\mathrm{N}$ foliar entre $30 \mathrm{e}$ $55 \mathrm{~g} \mathrm{~kg}^{-1}$ como valores adequados para o brócolo. Sendo assim, os teores de $\mathrm{N}$ foliar obtidos estão dentro da faixa considerada adequada.

O teor de potássio não foi influenciado significativamente pelos fatores estudados. Também não houve ajuste significativo da superfície de resposta. A ausência de variação significativa no teor de $\mathrm{K}$ foliar pode ser atribuída ao elevado teor de $\mathrm{K}$ no solo, conforme limites de interpretação para o nutriente no solo, citados por Raij et al. (1997). $\mathrm{O}$ teor médio observado de $\mathrm{K}$ na folha diagnóstica do brócolo foi de $16,8 \mathrm{~g}$ $\mathrm{kg}^{-1}$, teor que se encontra fora da faixa considerada adequada (20 a $40 \mathrm{~g} \mathrm{~kg}^{-1}$ ) por Trani \& Raij (1997) para brócolo.

A produtividade de inflorescências (PI) foi influenciada significativamente pela interação dos fatores doses de NK e espaçamento entre plantas. Houve ajuste significativo da superfície de resposta. Incrementos na PI foram obtidos aumentando-se a dose NK e diminuindo-se o espaçamento entre plantas. A máxima PI foi de 22,08 $\mathrm{tha}^{-1}$, que ocorreu no espaçamento de $0,2 \mathrm{~m}$ entre plantas com $150 \%$ da dose NK (315 $\mathrm{kg} \mathrm{ha}^{-1}$ de $\mathrm{N}$ e de $\mathrm{K}_{2} \mathrm{O}$ ), enquanto

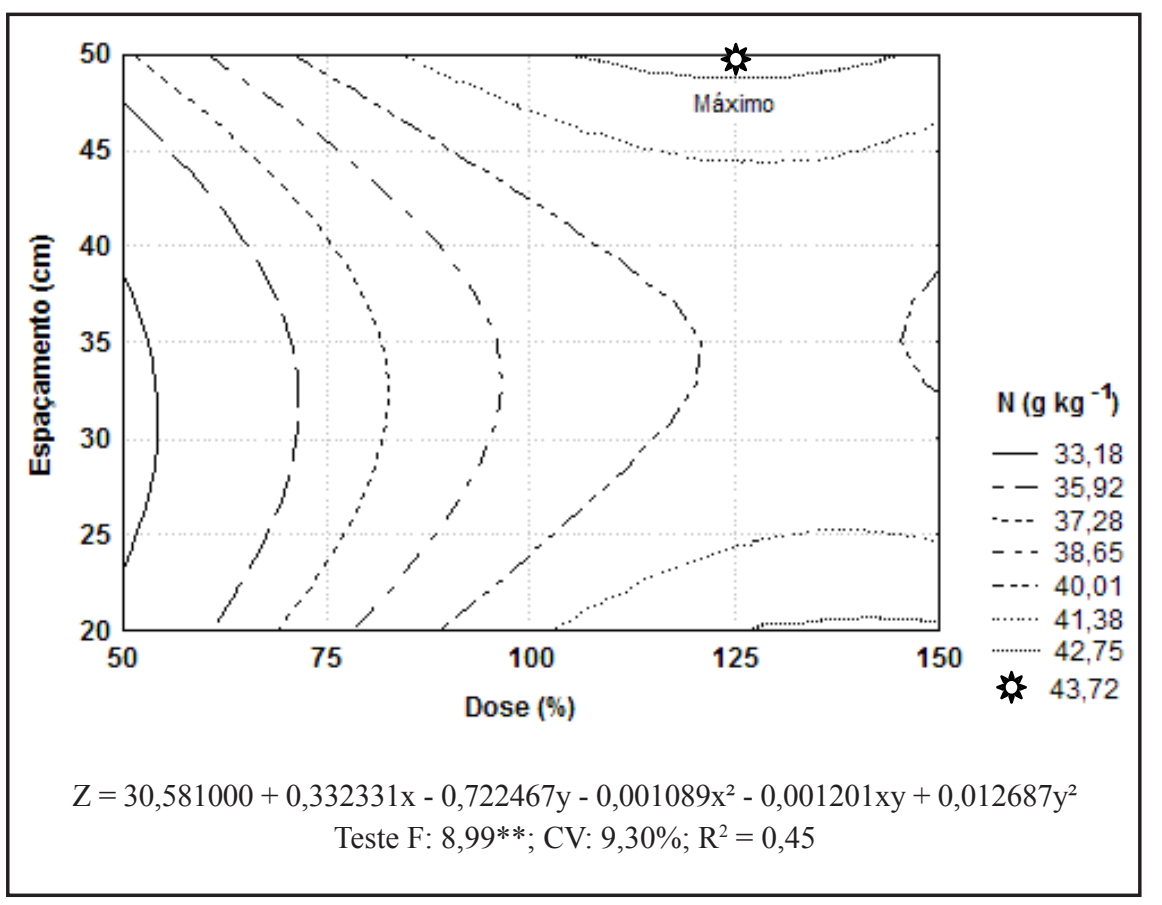

Figura 1. Isolinhas da superfície de resposta para teor $\left(\mathrm{g} \mathrm{kg}^{-1}\right)$ de nitrogênio $(\mathrm{N})$ em brócolos, em função do espaçamento entre plantas, na linha, e percentagem da dose $\mathrm{N}\left(210 \mathrm{~kg} \mathrm{ha}^{-1}\right) \mathrm{e}$ $\mathrm{K}_{2} \mathrm{O}\left(210 \mathrm{~kg} \mathrm{ha}^{-1}\right)$ (response surface for nitrogen (N) content $\left(\mathrm{g} \mathrm{kg}^{-1}\right)$ in broccoli, depending on the spacing between plants in row and percentage dose of $\mathrm{N}\left(210 \mathrm{~kg} \mathrm{ha}^{-1}\right)$ and $\mathrm{K}_{2} \mathrm{O}(210$ $\left.\mathrm{kg} \mathrm{ha}^{-1}\right)$ ). Jaboticabal, UNESP, 2010.

a menor PI $\left(13,44 \mathrm{t} \mathrm{ha}^{-1}\right)$ foi verificada com 0,5 m entre plantas e $150 \%$ da dose NK (Figura 2a). Adotando-se 0,2 m entre plantas, com o qual foi máxima a PI, e reduzindo-se a dose de NK para 262,5; 210,$0 ; 157,5$ e $105,0 \mathrm{~kg} \mathrm{ha}^{-1}$ de $\mathrm{N}$ e de $\mathrm{K}_{2} \mathrm{O}$, as PI foram 20,80; 19,09; $16,94 \mathrm{e}$ $14,36 \mathrm{t} \mathrm{ha}^{-1}$, respectivamente. Por outro lado, mantendo-se a dose de $150 \%$ da dose NK (315 kg ha-1 de $\mathrm{N}$ e de $\left.\mathrm{K}_{2} \mathrm{O}\right)$ e aumentando-se o espaçamento entre plantas para 0,$3 ; 0,4$ e $0,5 \mathrm{~m}$, também houve redução na PI, sendo de 18,98 ; 16,10 e $13,44 \mathrm{t} \mathrm{ha}^{-1}$, respectivamente (Figura 2a). Incrementos de PI como resultado do aumento na densidade de plantio também foram observados por Knaflewski \& Spizewski (2001). Esses autores obtiveram $8,5 \mathrm{tha}^{-1}$ de inflorescências na menor densidade de plantio (3 plantas $\mathrm{m}^{-2}$ ), enquanto na maior população $\left(10\right.$ plantas $\left.\mathrm{m}^{-2}\right)$, a produtividade foi de $14,3 \mathrm{tha}^{-1}$.

A produtividade de floretes (PF) foi influenciada significativamente tanto pelo fator doses NK quanto por espaçamento entre plantas. Houve ajuste significativo da superfície de resposta. A máxima PF foi de 17,09 $\mathrm{t} \mathrm{ha}^{-1}$, obtida com o mesmo espaçamento $(0,2 \mathrm{~m})$ e dose NK $\left(315,0 \mathrm{~kg} \mathrm{ha}^{-1} \mathrm{~N}-\mathrm{K}_{2} \mathrm{O}\right)$ em que a PI foi maximizada (Figura 2b). A máxima $P F$ correspondeu a 77,4\% da máxima PI. A diferença, cerca de $5 \mathrm{t}$, refere-se à parte da haste floral que não é utilizada pela indústria que comercializa brócolos congelados. A mínima PF, 9,58 tha ${ }^{-1}$, foi obtida com o maior espaçamento entre plantas $(0,5 \mathrm{~m})$ e menor dose NK $(105,0$ $\mathrm{kg} \mathrm{ha}^{-1} \mathrm{~N}-\mathrm{K}_{2} \mathrm{O}$ ) (Figura 2b), condição de cultivo que reduziu a $\mathrm{PF}$ em $44 \%$ da máxima.

$\mathrm{O}$ efeito do espaçamento entre plantas foi tão grande na PF que o aumento de apenas $10 \mathrm{~cm}$ entre plantas, mantendo-se a dose de $315 \mathrm{~kg} \mathrm{ha}^{-1}$ de $\mathrm{NK}_{2} \mathrm{O}$, causou redução de $13 \%$ na $\mathrm{PF}$. $\mathrm{O}$ resultado observado neste trabalho para o híbrido Mônaco, em resposta ao adensamento, corrobora o observado por Trevisan et al. (2003), que avaliando o brócolo 'Legacy', sob nove densidades de plantio resultantes da combinação de três espaçamentos entre linhas $(0,6 ; 0,8 \mathrm{e}$ $1,00 \mathrm{~m})$ e três espaçamentos entre plantas na fila $(0,3 ; 0,5$ e $0,7 \mathrm{~m})$, verificaram que maior produtividade foi obtida com maior população de plantas.

$\mathrm{O}$ rendimento industrial não foi influenciado significativamente pelos 


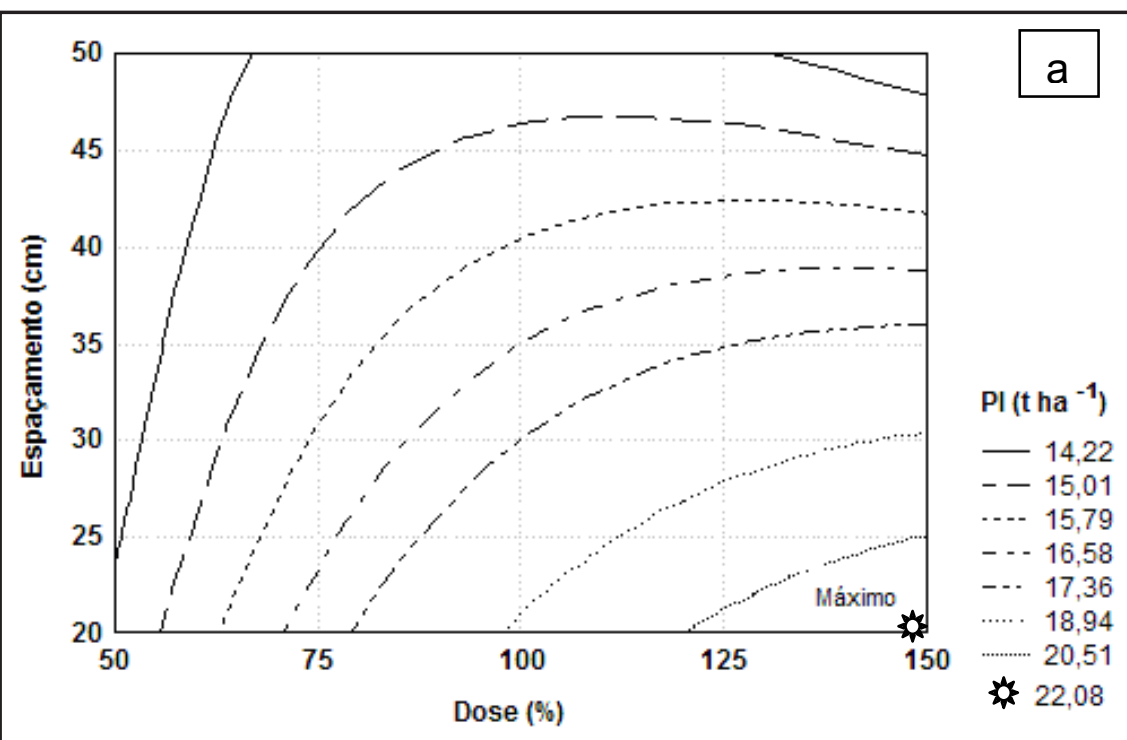

$Z=6,980760+0,198406 x+0,023810 y-0,000345 x^{2}-0,002602 x y+0,001211 y^{2}$ Teste F: $17,69^{* *} ; \mathrm{CV}: 12,62 \% ; \mathrm{R}^{2}=0,63$

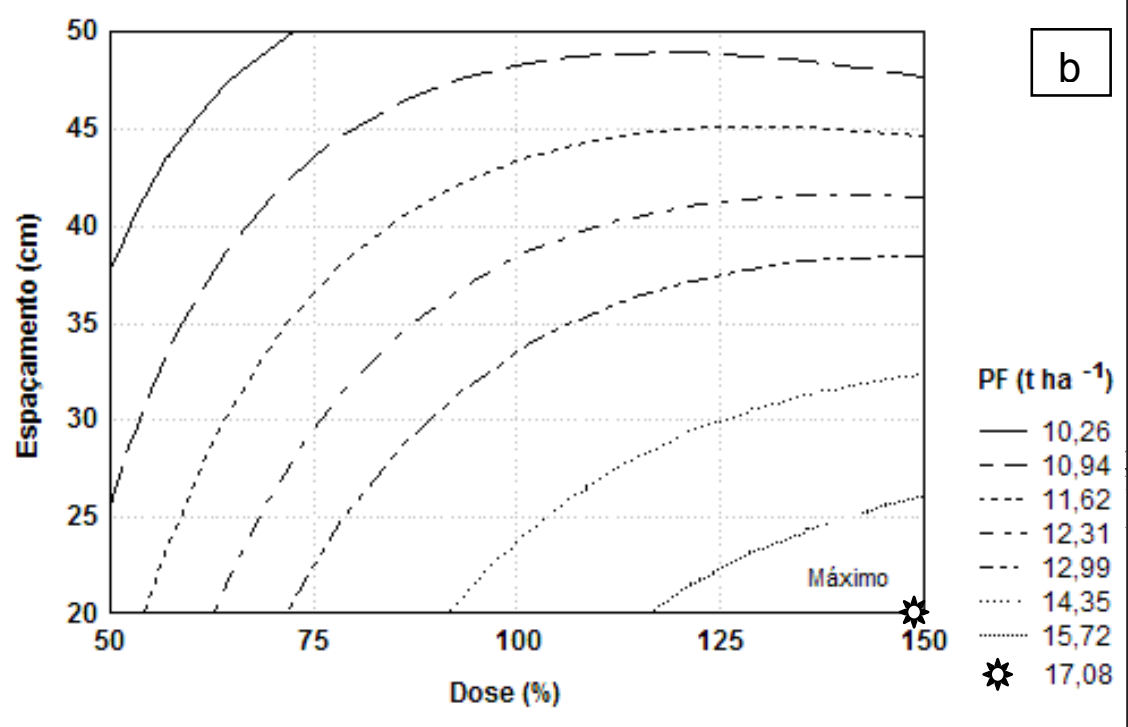

$Z=5,708011+0,147537 x+0,026342 y-0,000279 x^{2}-0,002602 x y+0,000025 y^{2}$ Teste F: $20,21 * * ; \mathrm{CV}: 12,89 \% ; \mathrm{R}^{2}=0,66$

Figura 2. Isolinhas da superfície de resposta para produtividade de inflorescências (PI) (a) e de floretes (PF) (b) ( $\left.\mathrm{t} \mathrm{ha}^{-1}\right)$ de brócolos, em função do espaçamento entre plantas na linha e percentagem da dose $\mathrm{N}\left(210 \mathrm{~kg} \mathrm{ha}^{-1}\right)$ e $\mathrm{K}_{2} \mathrm{O}\left(210 \mathrm{~kg} \mathrm{ha}^{-1}\right)$ (response surface for yield of inflorescences (PI) and florets (PF) of broccoli, as function of the spacing between plants in row and percentage dose of $\mathrm{N}\left(210 \mathrm{~kg} \mathrm{ha}^{-1}\right)$ and $\left.\mathrm{K}_{2} \mathrm{O}\left(210 \mathrm{~kg} \mathrm{ha}^{-1}\right)\right)$. Jaboticabal, UNESP, 2010.

fatores. Também não houve ajuste significativo da superfície de resposta para a interação dos fatores. $\mathrm{O}$ rendimento industrial médio foi de $77 \%$, com variação de 76,2 a $78,6 \%$ da produção de inflorescências.

A percentagem de floretes menores que $5 \mathrm{~cm}$ (PF5) foi influenciada significativamente somente pela adubação. cm (Figura 3a). Tal fato demonstra a forte associação entre inflorescências pequenas e floretes curtos.

A percentagem de floretes entre 5 e 7 cm (PF57), comprimento preferido pela indústria de congelados, foi influenciada significativamente pela dose NK e espaçamento entre plantas e houve ajuste significativo da superfície de resposta para interação dos fatores. A percentagem de floretes entre 5 e $7 \mathrm{~cm}$ foi grande. O mínimo de $43 \%$ foi obtido com $0,2 \mathrm{~m}$ entre plantas associado à menor dose de $\mathrm{NK}$, enquanto a percentagem máxima foi de $70 \%$ quando o espaçamento entre plantas foi de $0,4 \mathrm{~m}$ e as plantas receberam a maior dose de $\mathrm{N}_{\text {e }} \mathrm{K}_{2} \mathrm{O}, 315 \mathrm{~kg}$ ha $^{-1}$ (Figura 3b).

Nota-se que a dose de NK que maximizou a PF e a PF57 foi a mesma, mas com espaçamentos distintos, visto que a PF engloba todas as classes. Porém, ao contrário do que se observou na $\mathrm{PF}$, a economia de quase um terço da dose (105 kg ha ${ }^{-1} \mathrm{~N} \mathrm{e} \mathrm{K}_{2} \mathrm{O}$ ), de 315 para 210 $\mathrm{kg} \mathrm{ha}^{-1}$, mantendo-se o espaçamento de 0,4 m que maximizou a PF57, causou redução de apenas $2 \%$ no percentual máximo de floretes com comprimento entre 5 e $7 \mathrm{~cm}$, o que é interessante para o produtor.

Quanto mais próximas as plantas, aumentos na dose NK proporcionaram menores incrementos nos percentuais de floretes entre 5 e $7 \mathrm{~cm}$ de comprimento. Por exemplo, adotando-se $0,3 \mathrm{~m}$ entre plantas, a PF57 foi de $60 \%$ do total produzido quando adubado com 157,5 $\mathrm{kg} \mathrm{ha}^{-1}$ de $\mathrm{Ne} \mathrm{K}_{2} \mathrm{O}$ ( $75 \%$ da dose NK de referência, $210 \mathrm{~kg} \mathrm{ha}^{-1}$ ), mas quando foi utilizado $0,25 \mathrm{~m}$ entre plantas, para obter o mesmo percentual de floretes com 5 a $7 \mathrm{~cm}$, necessitou-se fornecer $262,5 \mathrm{~kg}$ ha $^{-1}$, o que equivale a $105 \mathrm{~kg} \mathrm{ha}^{-1}$ a mais de $\mathrm{N}$ e $\mathrm{K}_{2} \mathrm{O}$.

A percentagem de floretes entre $7 \mathrm{e}$ $9 \mathrm{~cm}$ de comprimento não foi influenciada significativamente pelos fatores avaliados e também não houve ajuste significativo da superfície de resposta para a interação dose $\mathrm{NK}_{2} \mathrm{O}$ e espaçamento entre plantas. A PF79 ajustou-se à equação polinomial de segundo grau em função da dose NK $(\mathrm{Y}=-10,365+$ $\left.0,448 x-0,0023 x^{2}, R^{2}=0,70 *\right)$. A PF79 foi maximizada ( $11 \%$ da PF) com 204 $\mathrm{kg} \mathrm{ha}^{-1}$ de $\mathrm{NK}_{2} \mathrm{O}$. Tanto a menor dose 


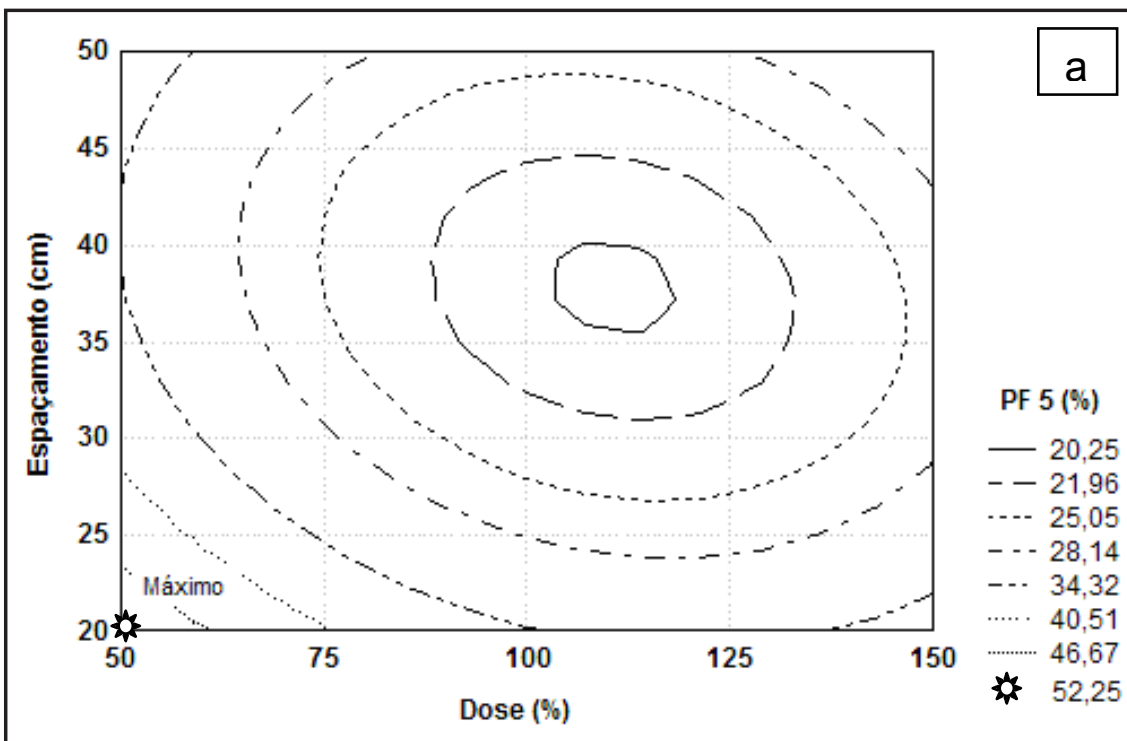

$Z=145,824354-1,026239 x-3,659251 y+0,003949 x^{2}+0,004053 x y+0,042494 y^{2}$

Teste F: $3,49 * * ; C V: 44,76 \%$; R2 = 0,51

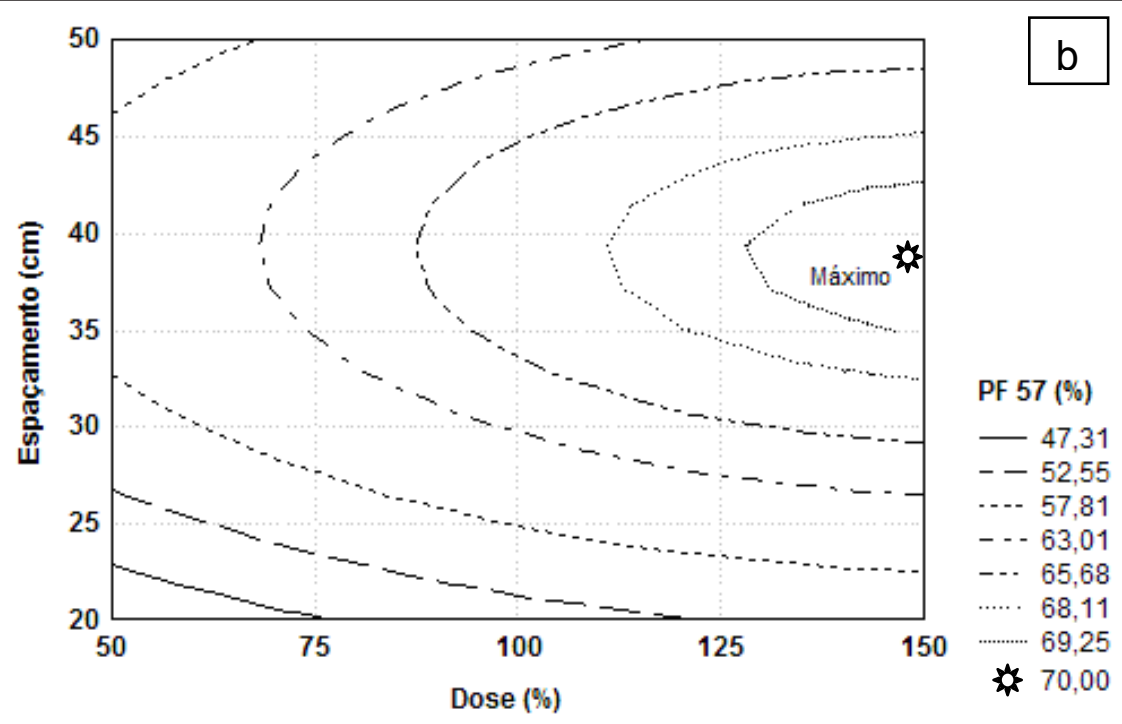

$Z=-23,639270+0,297840 x+3,618012 y-0,000872 x^{2}-0,000571 x y-0,045491 y^{2}$ Teste F: 4,37**; CV: 19,96\%; $\mathrm{R}^{2}=0,63$

Figura 3. Isolinhas da superfície de resposta para percentagem da produção total com floretes de brócolos menores que $5 \mathrm{~cm}$ de comprimento (PF5) (a) e de 5 a $7 \mathrm{~cm}$ (PF57) (b), em função do espaçamento entre plantas na linha e percentagem da dose $\mathrm{N}\left(210 \mathrm{~kg} \mathrm{ha}^{-1}\right)$ e $\mathrm{K}_{2} \mathrm{O}\left(210 \mathrm{~kg} \mathrm{ha}^{-1}\right)$ (response surface for percent of total production with broccoli florets smaller than $5 \mathrm{~cm}$ in length (PF5) (a) and 5-7 cm (PF57) (b) of broccoli, as function of the spacing between plants in row and percentage dose of $\mathrm{N}\left(210 \mathrm{~kg} \mathrm{ha}^{-1}\right)$ and $\mathrm{K}_{2} \mathrm{O}(210 \mathrm{~kg}$ ha $\left.^{-1}\right)$ ). Jaboticabal, UNESP, 2010.

$\left(105 \mathrm{~kg} \mathrm{ha}^{-1}\right)$ quanto a maior $(315 \mathrm{~kg}$ ha $\left.^{-1}\right)$ causaram reduções significativas na PF79, sendo $6 \%$ e $5 \%$, respectivamente.

A percentagem de floretes maiores que $9 \mathrm{~cm}$ somente foi influenciada significativamente pelo espaçamento entre plantas. Não foi verificado ajuste signi- $\mathrm{cm}$ não foram observados, enquanto no maior espaçamento $(0,5 \mathrm{~m})$ obteve-se o máximo de $1,5 \%$ da $P F$.

Com os resultados verificados, conclui-se que as maiores produtividades de inflorescências $\left(22,08\right.$ tha $\left.^{-1}\right)$ e de floretes $\left(17,09\right.$ t ha $\left.^{-1}\right)$ são obtidas com 0,2 $\mathrm{m}$ entre plantas e $315 \mathrm{~kg} \mathrm{ha}^{-1}$ de $\mathrm{N}$ e de $\mathrm{K}_{2} \mathrm{O}$; que os fatores espaçamento entre plantas e doses de NK não influenciam o rendimento industrial, que é de $77 \%$, e que do total produzido, a percentagem de floretes entre 5 e $7 \mathrm{~cm}$, comprimento preferido pela indústria de congelados, é maximizado em $70 \%$ da produtividade de floretes quando o brócolo é plantado com $0,4 \mathrm{~m}$ entre plantas e $315 \mathrm{~kg} \mathrm{ha}^{-1}$ de $\mathrm{NK}_{2} \mathrm{O}$.

\section{REFERÊNCIAS}

ARJONA DH; GREIG J. 1984. Study of the effect of four planting distances and four levels of nitrogen fertilization on yields, chlorophyll content and nitrate reductase activity in broccoli. Agronomia Colombiana 2: 71-81.

CRISP P; GRAY AR; ANGELL1 SM; SALTER PJ; AKEHURST J; SUTHERLAND RA. 1986. The effects of plant spacing on the breeding of broccoli from an expanded genetic base. Journal of Horticultural Science 61: 205-215.

DAVIS JM. 1998. Broccoli production guide for western North Carolina. Raleigh: Published by North Carolina Cooperative Extension Service. 3p.

EPSTEIN E; BLOOM AJ (trad. NUNES MET). 2006. Nutrição mineral de plantas: princípios e perspectivas. Londrina: Planta, $403 \mathrm{p}$.

EVERAARTS AP. 1994. Nitrogen fertilization and head rot in broccoli. Netherlands Journal of Agricultural Science 42: 195-201.

FRANCESCANGELI N; SANGIACOMO MA; MARTI H. 2006. Effects of plant density in broccoli on yield and radiation use efficiency. Scientia Horticulturae 110: 135-143.

GORSKI SF; ARMSTRONG DM. 1985. The influence of spacing and nitrogen rate on yield and hollow stem in broccoli. Research Circular 288: 16-18.

JOHNSON IT. 2002. Glucosinolates: bioavailability and importance to health. International Journal for Vitamin and Nutrition Research 72: 26-31.

KAHN BAB; EDELSON J; DAMICONE JP. 2004. Cole crop production (broccoli, cabbage, and cauliflower). Stillwater: Oklahoma State University. Division of Agricultural Sciences and Natural Resources, p. 6027/1-6027-7.

KNAFLEWSKI M; SPIZEWSKI T. 2001. Effect of plant density on the yield and quality of broccoli. Vegetable Crops Research Bulletin 55: 65-69.

KRISTAL AR; LAMPE JW. 2002. Brassica 
vegetables and prostate cancer risk: a review of the epidemiological evidence. Nutrition and Cancer 42: 1-9.

LeSTRANGE M; MAYBERRY KS; KIOKE ST.; VALENCIA J. 1996. Broccoli production in California. Oakland: University of California. Division of Agriculture and Natural Resources. 3p. (Publication, 7211).

LUENGO RFA; PARMAGNANI RM; PARENTE MR; LIMA MFBF. 2007. Tabela de composição nutricional das hortaliças. Disponível em: <http://cidadao.correioweb. com.br/hortalicas/tabelahortalicas.htm\#>. Acesso em: 13 fev

PASCHOLD PJ. 1986. Improvements in production make the cultivation of broccoli more interesting. Gartenbau 33: 113-114.

RAIJ B; CANTARELLA H; QUAGGIO JA; FURLANI AMC. 1997. Fósforo e potássio. In: RAIJ B; CANTARELLA H; QUAGGIO
JA; FURLANI AMC. Recomendações de adubação e calagem para o estado de São Paulo. Campinas: IAC, p.9-10. (Boletim técnico, 100).

SANDERS DC. 2001. Broccoli production. Raleigh: Published by the North Carolina Cooperative Extension Service, 1p.

SEABRA JÚNIOR. 2005. Influência de doses de nitrogênio e potássio na severidade à podridão negra e na produtividade de brócolis tipo inflorescencia única. Botucatu: UNESP-FCA. 81p. (Tese doutorado).

STEINKELLNER H; RABOT S; FREYWALD C; NOBIS E; SCHARF G; CHABICOVSKY M; KNASMULLER S; KASSIE F. 2001. Effects of cruciferous vegetables and their constituents on drug metabolizing enzymes involved in the bioactivation of DNA-reactive dietary carcinogens. Mutation Research 480481: 285-297.
TRANI PE; PASSOS FA; AZEVEDO FILHO JA; TAVARES M. 1997. Brócolos, couveflor e repolho. In: RAIJ B; CANTARELLA H; QUAGGIO JA; FURLANI AMC. Recomendações de adubação e calagem para o estado de São Paulo. Campinas: IAC, p.175. (Boletim técnico, 100).

TRANI PE; RAIJ B. 1997. Hortaliças. In: RAIJ B; CANTARELLAH; QUAGGIO JA; FURLANI AMC. Recomendações de adubação e calagem para o estado de São Paulo. Campinas: IAC, p.163. (Boletim técnico, 100).

TREVISAN BG; TREVISAN BGN; MARTINS GAK; LOPES SJ; LOVATO DS; BACKES GM. 2003. Rendimento de brócolis Legacy sob nove densidades de plantio. In: MOSTRA DE INICIAÇÃO CIENTÍFICA, 2; JORNADA DE PÓS GRADUÇÃO, PESQUISA E EXTENSÃO, 2, Bagé-RS. Anais ... Bagé: URCAMP, p.93. 